\title{
Terahertz pulse imaging in archaeology
}

Article

Accepted Version

Jackson, B., Labaune, J., Bailleul-Lesuer, R., D'alessandro, L., Whyte, A., Bowen, J., Menu, M. and Mourou, G. (2015) Terahertz pulse imaging in archaeology. Frontiers of Optoelectronics, 8 (1). pp. 81-92. ISSN 2095-2767 doi: https://doi.org/10.1007/s12200-014-0446-y Available at https://centaur.reading.ac.uk/37216/

It is advisable to refer to the publisher's version if you intend to cite from the work. See Guidance on citing.

To link to this article DOI: http://dx.doi.org/10.1007/s12200-014-0446-y

Publisher: Springer

All outputs in CentAUR are protected by Intellectual Property Rights law, including copyright law. Copyright and IPR is retained by the creators or other copyright holders. Terms and conditions for use of this material are defined in the End User Agreement.

\section{www.reading.ac.uk/centaur}

\section{CentAUR}

Central Archive at the University of Reading

Reading's research outputs online 
J. Bianca JACKSON et al. Terahertz pulse imaging in Archaeology

Front. Optoelectron. (2014)

DOI

1 RESEARCH ARTICLE

\title{
Terahertz Pulse Imaging in Archaeology
}

\author{
J. Bianca JACKSON $(\varangle)^{1,3}$, Julien LABAUNE $^{1}$, Rozenn BAILLEUL-LESUER $^{2}$, Laura D'AL
Alison WHYTE ${ }^{2}$, John BOWEN ${ }^{3}$, Michel MENU $^{4}$, Gerard MOUROU $^{1}$ \\ 1 Institute de la Lumière Extrême, Ecole Polytechnique, Palaiseau FR \\ 2 Oriental Institute, University of Chicago, Chicago, IL USA \\ 3 School of Systems Engineering, University of Reading, Reading UK \\ 4 Centre de Recherche et de Restauration des Musées de France, Paris, FR
}

(C) Higher Education Press and Springer-Verlag Berlin Heidelberg 2014

\begin{abstract}
The work presented in this article was performed at the Oriental Institute at the University of Chicago, on objects from their permanent collection: an ancient Egyptian bird mummy and three ancient Sumerian corroded copper-alloy objects. We used a portable, fiber-coupled terahertz time-domain spectroscopic imaging system, which allowed us to measure specimens in both transmission and reflection geometry, and present time- and frequency-based image modes. The results confirm earlier evidence that terahertz imaging can provide complementary information to that obtainable from x-ray CT scans of mummies, giving better visualisation of low density regions. In addition, we demonstrate that terahertz imaging can distinguish mineralized layers in metal artifacts.
\end{abstract}

Keywords terahertz, time-domain imaging, spectroscopy, non-destructive evaluation, archaeology

\section{Introduction}

Terahertz pulse imaging and spectroscopy (TPIS) is emerging as a nondestructive-evaluation tool of high growth potential within the fields of art conservation, historical architecture conservation, and archeology [1]. In particular with mummies, TPIS has been explored as an alternative - or complement - to X-ray imaging techniques [2,3]. It is the combination of material characterization, time of flight imaging, and the penetration of optically opaque materials that gives rise to applications for subsurface imaging of many culturally significant objects. Moreover, the variety and adaptability of the many electronic, optical, and hybrid terahertz sources allow for versatile approaches to measurement [4-6]. Spatial resolution for free-space terahertz measurements can be scaled from tens of micrometers to several millimeters, providing the possibility of taking measurements without sample extraction, in situ, and in the field [7]. Lastly, moderate exposure to

Received May 26, 2014; accepted

E-mail: jbiancajackson@gmail.com 
terahertz radiation poses significantly less long-term risk [8,9] to the molecular stability of the historical artifact and to humans than X-rays, UV or visible radiation, because it is non-ionizing and produces low thermal heating. Therefore, terahertz technology provides a non-ionizing, noninvasive, noncontact, nondestructive toolset [10] for the examination of unique and priceless objects [11].

The terahertz (THz) region is possibly the least understood and most complicated part of the electromagnetic spectrum The THz region has been typically defined as being between $30 \mu \mathrm{m}$ and $3 \mathrm{~mm}$ in wavelength, thus putting its scale on the border between the microscopic and macroscopic world. At frequencies between 0.1 and 10 trillion $\left(10^{12}\right)$ cycles per second, the terahertz regime overlaps with both the microwave and far infrared regions of the spectrum. The terahertz regime also corresponds to photon energies between 0.4 and $40 \mathrm{meV}$. Lower frequency microwave radiation has lower photon energy, therefore the photons cannot be measured directly, only collectively by the electrical bias they induce in a detector. On the other hand, infrared radiation is optical, since its photon energy is large enough that individual photons can be directly measured. Thus, terahertz radiation uniquely straddles the worlds of electronics and optics. Over the last two decades, means of producing and detecting sub-picosecond, broadband pulses of $\mathrm{THz}$ radiation by integrating optoelectronic devices with ultrafast optical lasers have sparked many new forms of research-including time-domain terahertz spectroscopy and imaging. As the terahertz technology gap is filled by diverse and practical devices, the number of $\mathrm{THz}$ applications constantly increases, including those developed for the chemical-mapping of pharmaceuticals, the non-destructive evaluation of space shuttle foam, people-safe security imaging, and atmospheric-chemical species monitoring [12,13].

Our TPIS technique is based on time-domain spectroscopy. The terahertz pulse is measured as a transient electric field, which can be directly transformed into the amplitude and phase spectra of the pulse. When the $\mathrm{THz}$ pulse is transmitted through an object of low electrical conductivity (thus relatively low absorption), compositional changes - which include refractive index and optical density - can result in changes of the amplitude, shape and propagation time of the $\mathrm{THz}$ pulse. By scanning the object, this can be exploited to image the lateral and axial spatial features buried beneath the visibly opaque surface of the object. While the wide bandwidth of the terahertz pulses can aid in spectroscopically discriminating between buried materials that exhibit different terahertz-refractive-index spectra, the short-time-duration nature of terahertz pulses can also help isolate and distinguish depth information from different interfaces within an object.

In the course of our study, we were permitted access to the conservation laboratory of the Oriental Institute at the University of Chicago to examine several archeological artifacts from their collection. The first is an Egyptian neonate bird mummy (OIM E9164, Fig. 1), which was to be included in a project to image several dozen bird mummies using X-ray computed tomography (XRCT) [14]. Challenges of such an endeavor included protecting the fragile objects from physical disturbance and fluctuations in humidity. Special packaging and precise coordination of transport to the medical imaging facilities were essential to the XRCT experiments. In contrast, the availability of portable THz-TDS systems enabled our measurements to be performed in the well-controlled environment of the conservation laboratory.

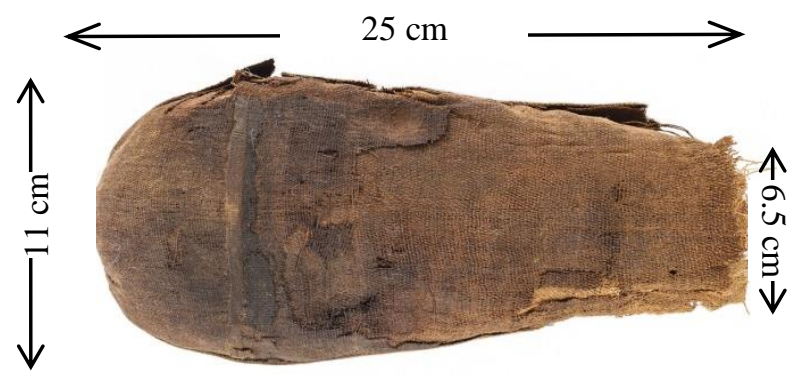

Fig. 1 Photograph of ancient Egyptian bird mummy (OIM E9164) [15].

Among the other selections we studied were corroded copper-alloy (most likely bronze) artifacts from Ancient Sumer (now Iraq), with special attention toward a $5 \mathrm{~cm}$ fragment from an unregistered object (Fig. 2) and two cups (Fig. 3). For many archeological artifacts made of bronze or other copper-based alloys, the 
66

67

68

69

70

71

72

73

corrosion process can produce multilayer oxide structures (Fig. 4) - such as copper + cuprite + copper trihydroxy chloride minerals + malachite + azurite - which through time and environmental exposure can entirely convert the metallic material to stratified, composite layers of dielectric minerals [16,17]. This particular application for TPIS is dependent on the ability of terahertz waves to distinguish dielectric corrosion products from their metallic source material by their transmissivity and reflectivity. Previous work has been done to find defects beneath the coating on metal surfaces [18,19], however not much THz research has been conducted to study the specific corrosion layers themselves. Terahertz pulses have the potential to spectroscopically identify and spatially distinguish each layer.

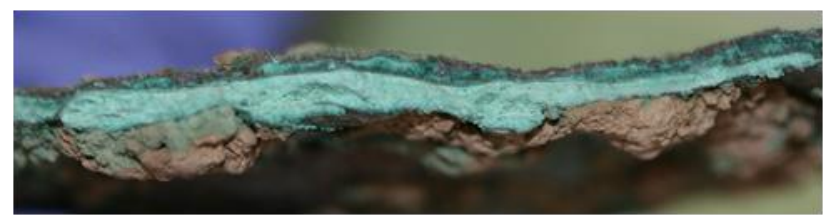

Fig. 2 Photo of corroded copper-alloy fragment cross-section [17].

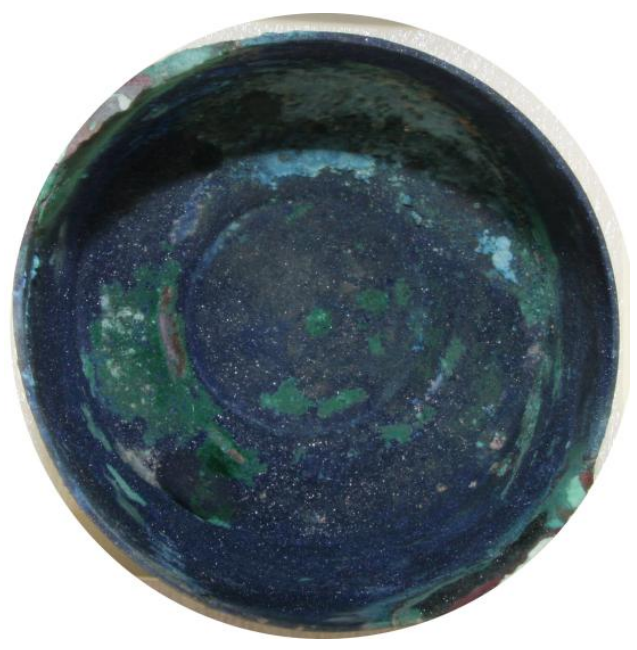

(a)

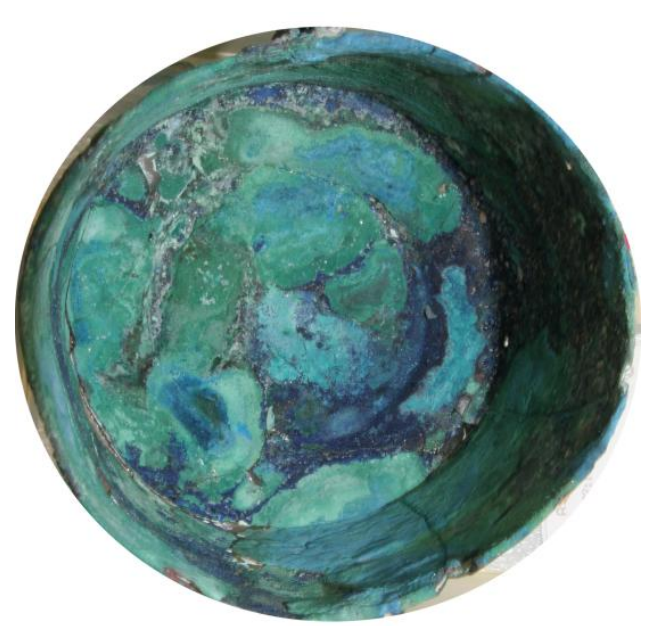

(b)

Fig. 3 Photographs of corroded copper-alloy a) cup A (OIM A11280A, $12 \mathrm{~cm}$ diameter) and cup B (OIM A11399A, $10 \mathrm{~cm}$ diameter) [17]. 


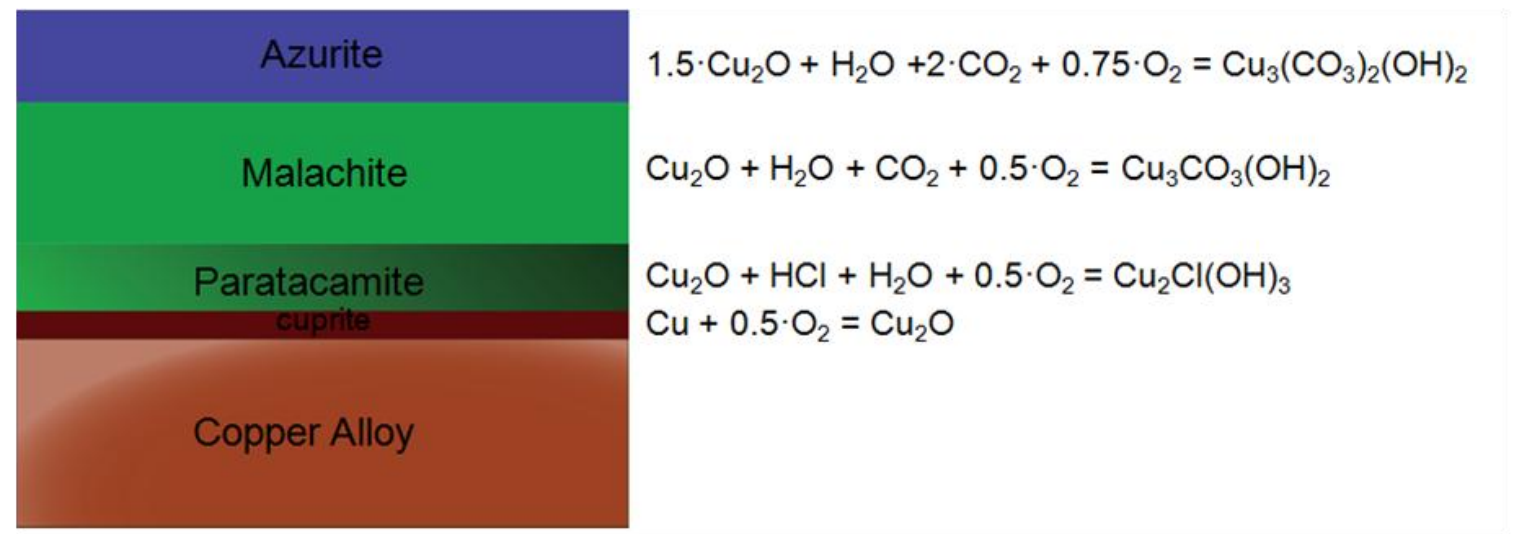

Fig. 4 Diagram of typical copper-based corrosion layers.

\section{Experimental methods}

78 Our time-domain terahertz imaging system consists of computer-controlled, motorized translation stages and the Picometrix T-Ray 4000 (TR4K) commercial THz time-domain spectrometer. The modelocked, two-stage, amplified, Ytterbium fiber laser operates with a center frequency near $1064 \mathrm{~nm}$, a $100 \mathrm{fs}$ pulse width, a 50 $\mathrm{MHz}$ repetition rate and a maximum output power of $400 \mathrm{~mW}$.

The terahertz pulses were generated and then propagated through free-space using a biased, photoconductive switch antenna, consisting of a photosensitive low-temperature grown gallium arsenide semiconductor with two metal electrodes deposited on its surface. The antenna is illuminated at normal incidence by the ultrafast laser pulse, thus generating electron-hole pairs into the semiconductor. A voltage bias is applied to the electrodes to generate a photocurrent. The free-space terahertz electromagnetic field emanating from the antennas is proportional to the rapid change in the photocurrent - the sub-mechanisms of which determine the $\mathrm{THz}$ pulse's duration and bandwidth. A second photoconductive antenna is used as the terahertz receiver. The optical pulse generates photocarriers in the receiver by the same photoexcitation mechanism as when the emitter is illuminated. However in this case, the incident electric field of the $\mathrm{THz}$ pulse causes a time-varying potential to develop across the receiver, thus serving as an applied voltage bias that induces a transient photocurrent, which is amplified and measured as an electrical signal by using a data acquisition board and computer.

The development of compact, fiber-coupled THz-TDS systems, such as the TR4K, has several benefits which were essential to the advancement of terahertz applications in art and archeology. As a general rule, cultural heritage conservators aim toward minimizing the disturbance of the objects under investigation. The optical components for the compact spectrometer are contained within an easy to transport (size and weight) box. This allows for lower complication on-site or field measurements. The fiber-coupled THz antennas permit rapid modification of the measurement geometry from transmission (Fig. 5a) to reflection (Fig. 5b), and vice versa. Additionally, it makes it possible for the object to remain fixed and secure during the scans, and facilitates the measurement of large objects in the transmission geometry. 


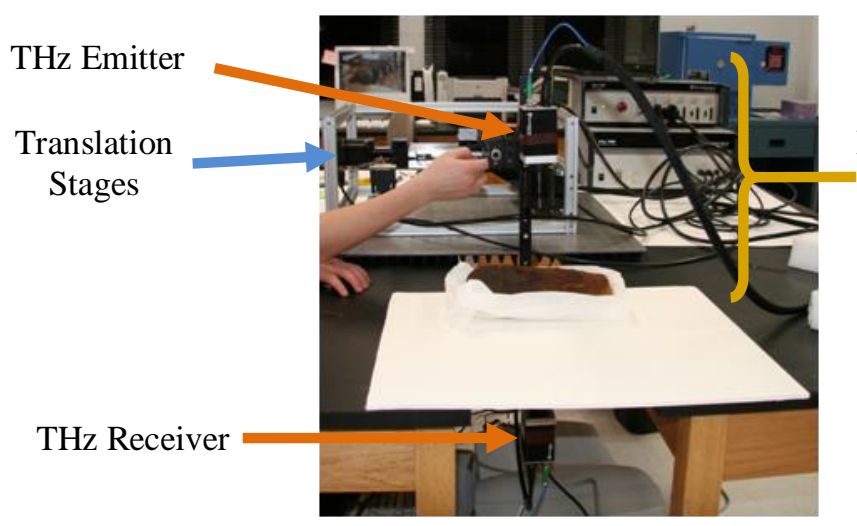

(a)

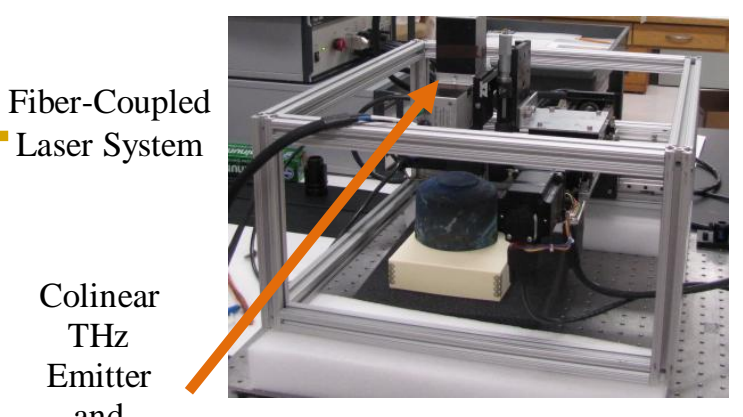

and

Receiver

(b)

\section{3}

Fig. 5 Photographs of the (a) transmission [15] and (b) reflection setups.

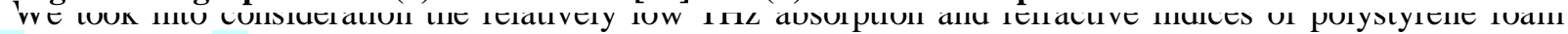
[20] and paper [21] and utilized a foamcore support (Fig. 5a), which could permit us to securely scan the top and bottom of the extremely fragile mummy in transmission with minimal consequence to the signal. Figure $6 \mathrm{a}$ shows $\mathrm{THz}$ time-domain signals through the ambient environment and the foam support. The terahertz spectra (Fig. 6b) are obtained by performing a fast Fourier transform of the time domain signals. The refractive index and absorption of the foamcore (Fig. 6c) were $\sim 20 \%$ and $\sim 1500 \%$ higher than expected, respectively. This may be due to the thickness, type and coating of the board's paper shell. For future experiments, a several millimeter thick sheet of uncoated, un-sheathed polystyrene, or polymethyacrylimide [22], would provide equally rigid support with less signal loss.

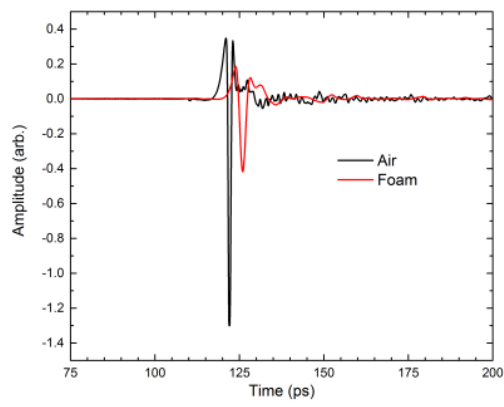

(a)

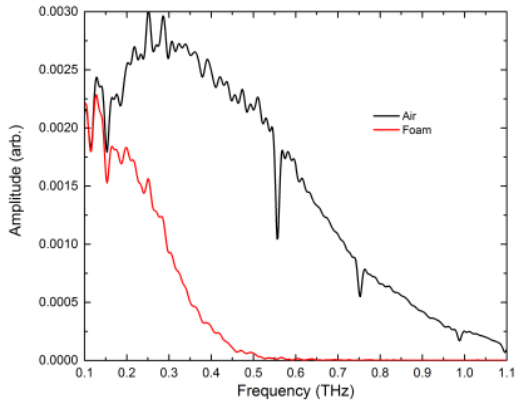

(b)

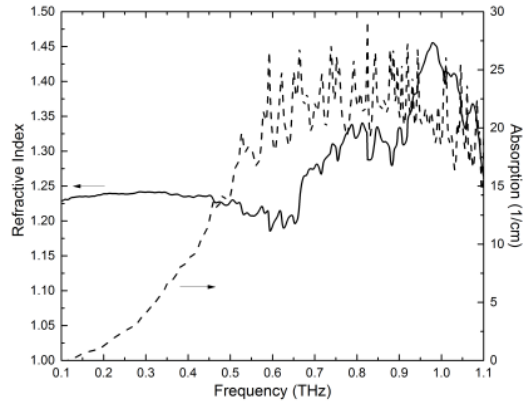

(c)

Fig. 6 Air and foam support reference (a) time domain signal, (b) transmission spectra and (c) refractive index and absorption spectra for the foam support.

\section{Discussion}

\subsection{Terahertz pulse imaging of a bird mummy}

Transmission through the thicker, upper region of the mummy was too small to discern a signal through the baseline noise, so we only scanned the lower $80 \%$. The two dimensional scans of the middle and lower regions can be seen in Fig. 7, and were calculated using the amplitude of the minimum peak for each pixel. The spatial resolution, or ability to see detail in the image, was negatively impacted by decreased bandwidth of the signal, as well as the changing spot size of the Gaussian beam through the bird. For the torso region, there is very strong absorption loss where the skeleton and desiccated organ tissue would be (black). For the leg region, the flesh and bone are less dense, resulting in better signal to noise and more distinct features. The shape of what 
appear to be the claws (although probably tibio-tarsal joints), however, is the most impressive feature of this image.

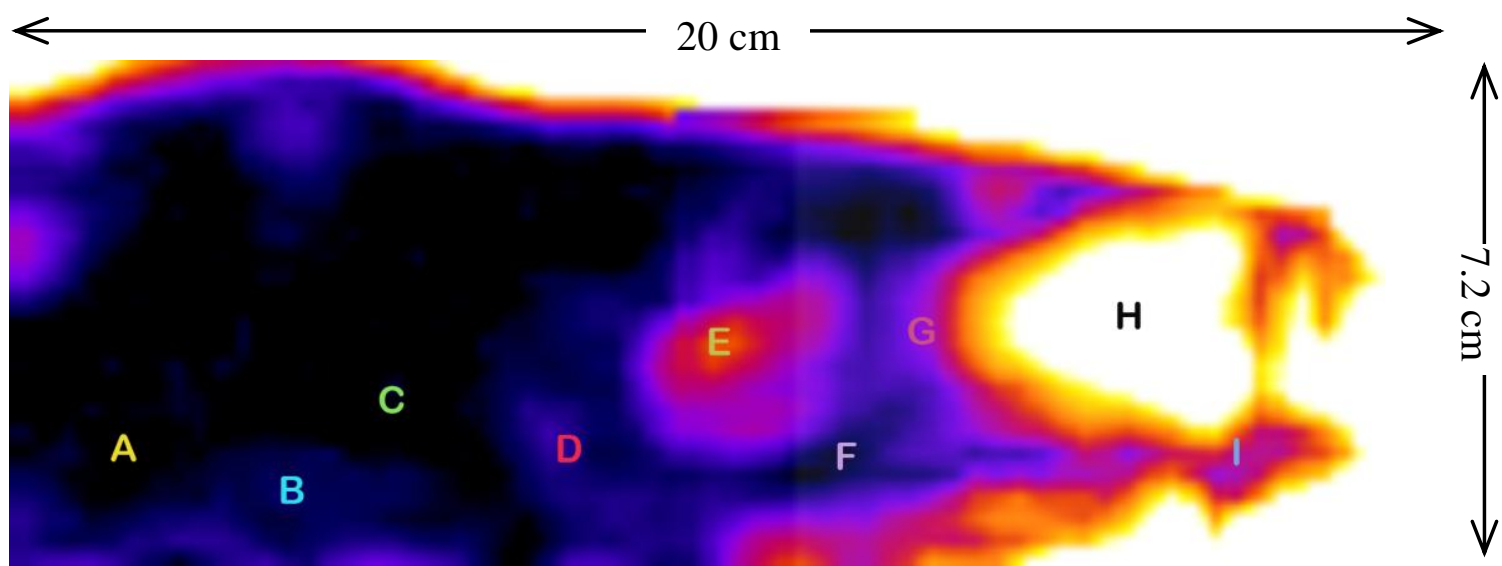

Figure 7: Terahertz transmission image of bird mummy \{Color scale: white/yellow $=$ higher transmission, black/violet $=$ lower transmission $\}$

Figure 8 shows exemplary signals through the middle region, or torso, and lower region, or legs, of the bird mummy, designated by the letters A-H in Fig. 7. The multiple peaks, seen in Fig. 8a and Fig. 8b, are a result of internal reflections of the $\mathrm{THz}$ pulse as it interacts with the textile wrap, desiccated flesh and bones of the bird. Note the one order of magnitude difference in transmission between the torso region and the leg region. The spectra in Fig. 8c also show significant signal loss above $0.2 \mathrm{THz}$, even in the "empty" region $\mathrm{H}$, which suggests the loss is most likely due to the electric field being scattered by the weave and layers of the cloth wrapping [23]. Fortunately, in the range of 0.1 to $0.2 \mathrm{THz}$, the attenuation of the foam support is comparable to air.

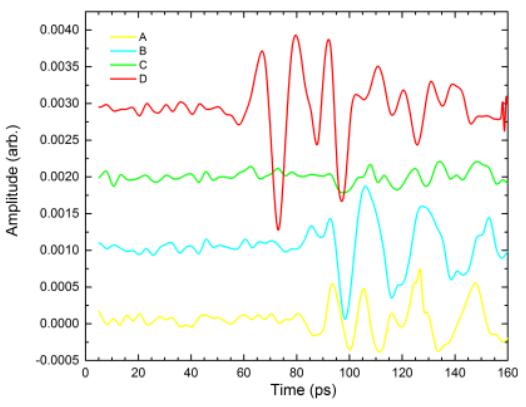

(a)

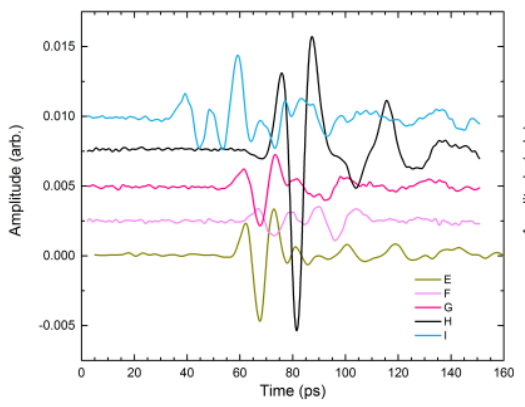

(b)

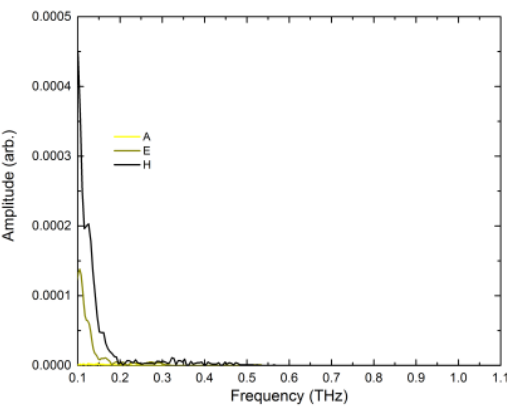

(c)

Fig. 8 Select (offset) time-domain waveforms from (a ) torso region and (b) leg region; (c) spectral waveforms from bird mummy transmission signals.

There are few published comparisons of mummies imaged using both X-rays and terahertz radiation. Previously, Öhrström et al also used terahertz transmission imaging to view the contents of a mummified human hand and fish [2]. They demonstrated that with TPIS it was possible to recognize differences in the desiccated flesh and that it adds a temporal aspect that does not exist in other techniques, despite TPIS having coarser resolution than XRCT. Fukunaga et al also confirmed that terahertz imaging may complement X-ray images when materials with low radiographic density and contrast are being investigated [3]. From the XRCT images taken on this bird mummy (Fig. 9), we can confirm this with significance. It is likely that since the bird was young at the time of its death, its bones were soft, resulting in low-density calcification. As a result, $\mathrm{THz}$ provides a much better visualization of the leg region of the bird than X-rays, as applied in this particular CT- 


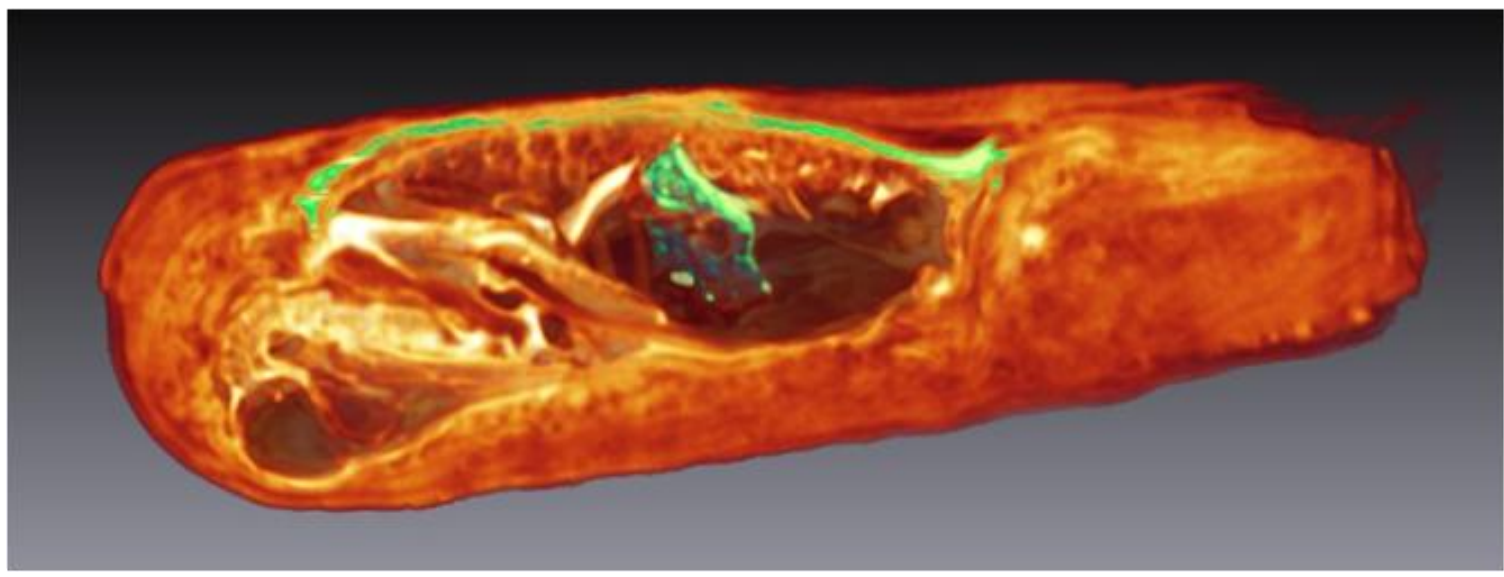

Fig. 9 X-ray CT scan of Egyptian bird mummy E9164 [14]. \{Courtesy of Charles Pelizzari and Christian Wietholt

\subsection{Corroded copper-alloy fragment}

147 A copper alloy fragment from an Early Dynastic ( 2000 BC) Sumerian unregistered object excavated from the 148 Tell Agrab settlement of the Diyala Valley in Iraq (Fig. 2) was examined to determine whether it is possible to distinguish the metallic and mineralized stratified corrosion layers. Figure 10a shows an image of the surface of the fragment, calculated using the peak to peak amplitude of each pixel. The brightest areas are where the surface of the fragment is still metallic, due to the high reflectivity. The pink line indicates the location of the slice, seen as the cross-sectional b-scan in Fig. 10b. Figure 10c shows the time-domain signatures of select point measurements. The outer crust is composed of calcified dirt, possibly tin and copper corrosion products. It is possible, however, to observe some significant reflectivity from the near-surface interfaces.

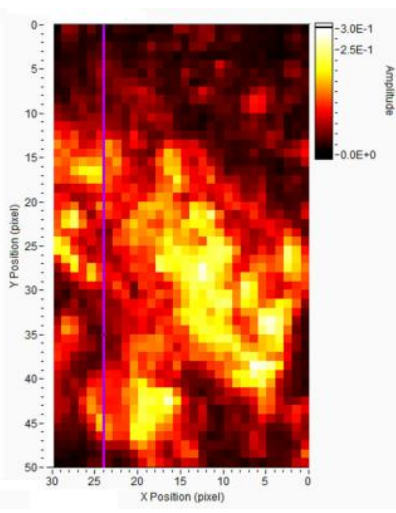

(a)

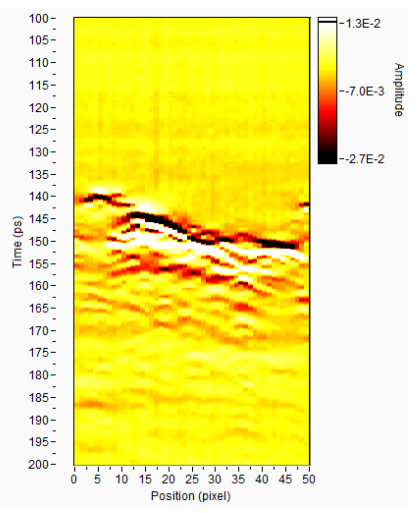

(b)

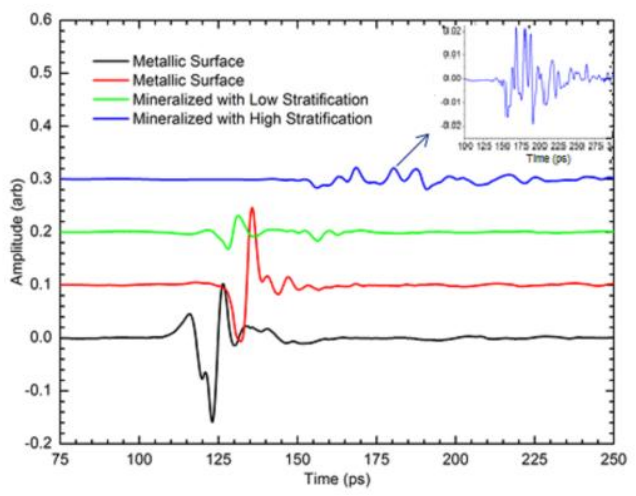

(c)

Fig. 10 (a) Peak to peak amplitude terahertz reflection image, (b) cross-sectional b-scan image, and (c) select time-domain signals (offset) [17]. 
Two Sumerian copper alloy cups excavated from the ancient city of Eshnunna-modern-day Tell Asmar in the Diyala Valley - were also investigated (Fig. 3). Cup A was covered by dark blue corrosion, presumably azurite crystals with some dark green areas, presumably malachite. The transmission through the base of cup A was at the signal noise floor, therefore the subsurface was still entirely metallic (Figure 11a). Cup B was covered in aqua colored corrosion, an admixture of azurite and malachite, with several regions of dark blue and green. Much of the base of cup B is completely mineralized. The black region of the terahertz transmission measurement in Figure 11b indicates that approximately $13 \%$ of the subsurface area has transmission approaching, but not at, the noise floor, unlike with cup A.

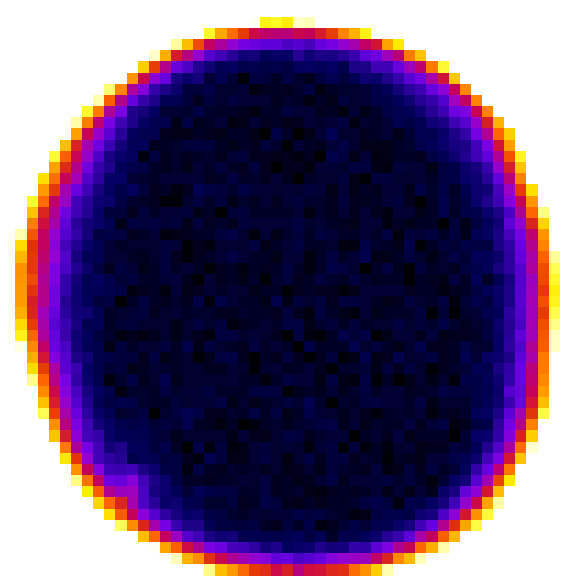

(a)

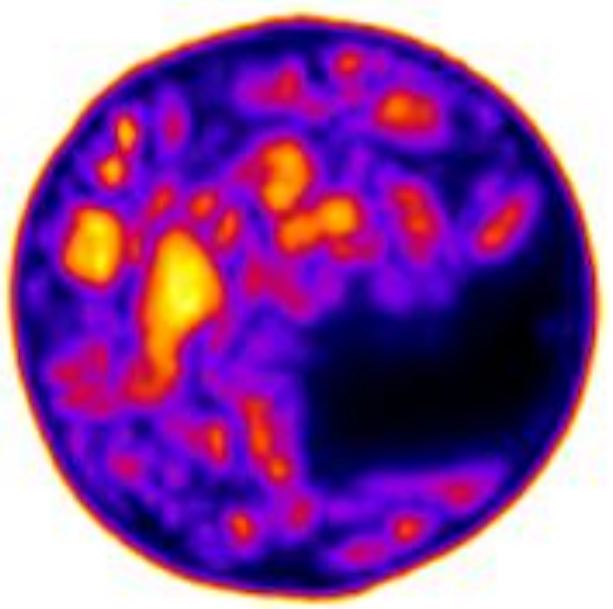

(b)

Fig. 11 Transmission images calculated using THz pulse minimum peak amplitude for a) cup A and b) cup B. \{Color scale, same: White/yellow $=$ high transmission, black/violet $=$ low transmission $\}$

First, we analyzed the minimum peak time-of-flight of both cups. Since no terahertz signal transmitted through cup A, the image of the minimum peak time was random, as seen in Fig. 12a. On the other hand for cup B (Fig. 12b), the minimum peak times were locally ordered and fall within a 10 ps time window. This helps to discount the possibility that any dominant pulse in that temporal region is an artificial product of the denoising process, as we would expect it to be random as well. Interestingly, there is not a direct correlation between the peak amplitude and time-of-flight; both regions with the highest and lowest peak amplitudes also have the longest time-of-flights. Additionally, the rim shape of the cup base has no impact on the transmitted 


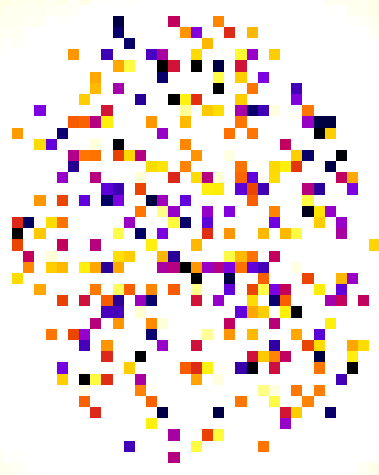

(a)

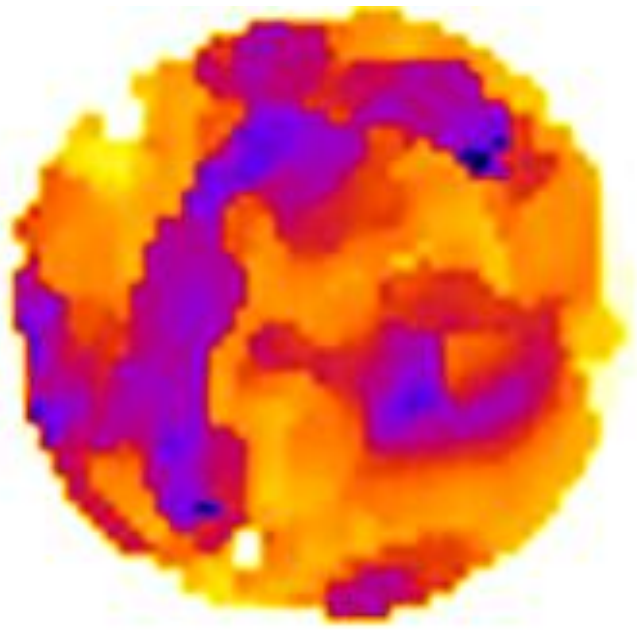

(b)

Fig. 12 Minimum peak time-of-flight for transmission signals through a) cup A and b) cup B. \{Color scale, same: white/yellow $=$ shortest time-of-flight $[132 \mathrm{ps}]$, black/violet = longest time-of-flight $[142 \mathrm{ps}]\}$

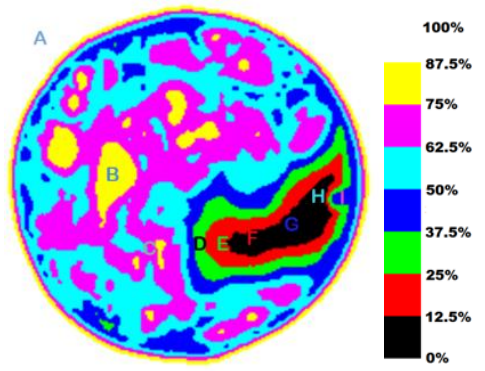

(a)

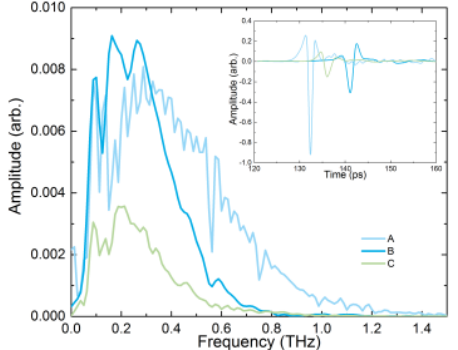

(b)

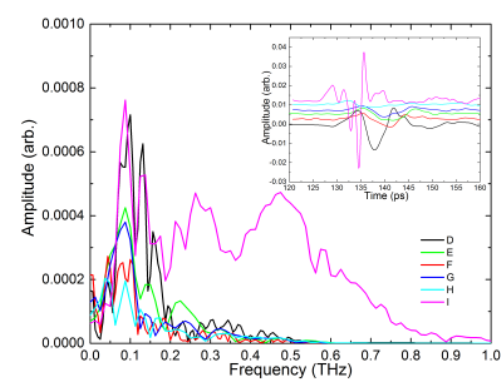

(c)

Fig. 13 a) Transmission factor, $\eta$, map of cup $B$; b) and c) select frequency- and time-domain (inset) waveforms.

Figure 13a shows a discrete color map of cup B's transmission factor, defined as:

$$
\eta=100\left[\frac{\log \left(E_{j} / E_{\min }\right)}{\log \left(E_{\max } / E_{\min }\right)}\right]
$$

178 where $E_{j}$ is the peak to peak amplitude of the time signal for an individual pixel, normalized by $E_{\text {min }}$, the peak to peak amplitude of the smallest pixel signal, and $E_{\max }$ is the peak to peak amplitude of the largest pixel signal (specifically, an air reference). For $\eta$ greater than $50 \%$ the pattern is consistent with the minimum peak amplitude image in Figure 11b. However below 50\%, the erstwhile black region now has four levels of intensity with the least transmissive region being reduced to an area approximately $9 \mathrm{~mm}$ wide and $36 \mathrm{~mm}$ long. The compositional nature of this region is not obvious, although it is probable that this smaller black region describes the location of the only remaining metallic subsurface.

We used Coif4 wavelets with 4 levels to denoise the time-domain waveforms and extract the transmitted signal, shown in Fig. 13b and Fig. 13c. Despite an order of magnitude difference in transmission between the 
wholly-mineralized and partially-mineralized region, there are at least two plausible explanations for the presence of $\mathrm{THz}$ signal in the black region of Fig. 13a. The first possibility is that at least part of that subsurface region is non-transmissive metal, with the full-width, Gaussian-beam diameter exceeding that surface area, resulting in lower frequencies leaking through the surrounding dielectric areas. This is consistent with the "concentric" shape of the blue, red and green regions around the black area, and with the narrow bandwidth, low-central frequency spectra selected as D-H in Fig. 13c.

On the other hand, it is also plausible that the black region comprises a remnant metallic layer of total thickness less than the skin depth of copper through which the terahertz pulse is propagating. If the skin depth of copper is given by [24]:

where $v$ is the terahertz frequency, then the total thickness of any remnant metal must be less than $75 \mathrm{~nm}$, since there is signal up to $0.6 \mathrm{THz}$. The likelihood of such a fine layer of copper is worth considering and intriguing, given the totality of the cup's oxidation, and the frequency independent attenuation of metal.

The bases of both cups (Fig. 14) were also measured in reflection. Figure 15a shows the image of cup A, calculated using the minimum peak amplitude of the reflected terahertz pulse. There was significant loss around the edges, due to the increased curvature; however, the reflectivity of the surface is very sensitive to the thickness and number of corrosive layers. Figure $15 b$ shows the $b$-scan - or cross-sectional signal amplitude image, with vertical axis corresponding to time and the horizontal axis corresponding to the space - of the slice denoted by the green line in Fig. 15a. This cross-section shows that in the violet areas, there are only two large reflections - one on the mineral surface and one on the metal surface. In the orange area, extra lines appear in the cross-section between the two strongest reflections, suggesting one or two different corrosion layers, whereas, in the yellow area, the reflections are the weakest and the cross-section shows that the yellow and white area is highly stratified.

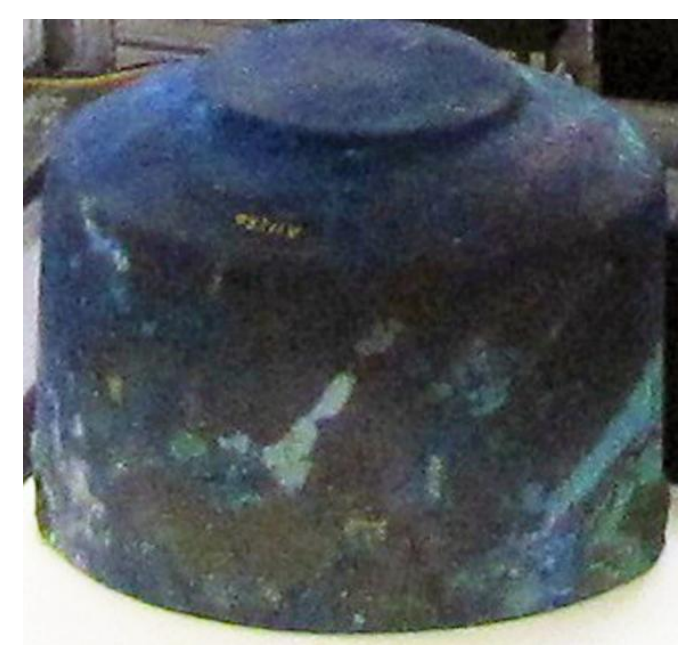

(a)

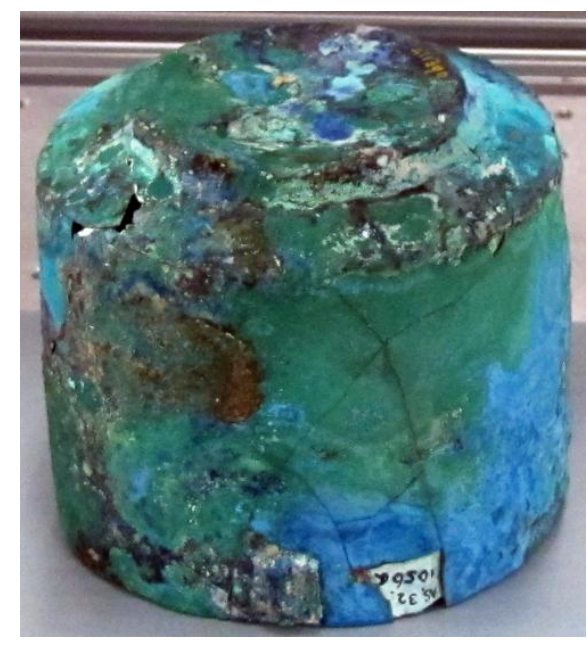

(b)

Fig. 14 Photographs of the bottom sides of a) cup A and b) cup B. 


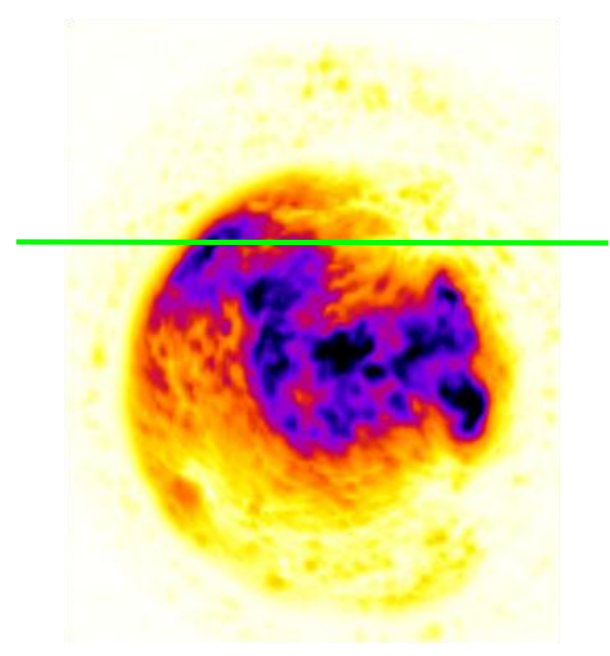

(a)

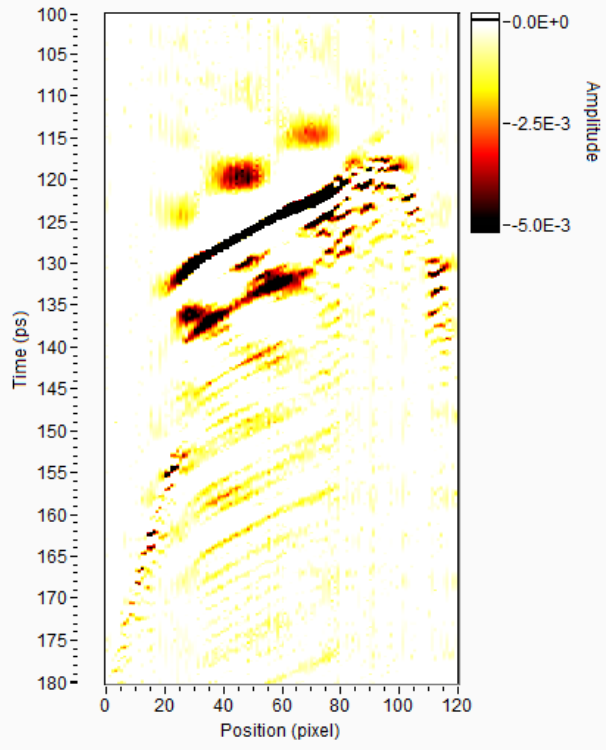

(b)

Fig. 15 a) Terahertz pulse reflection minimum peak image of cup $A$ \{Color scale: White/yellow = low reflection, black/violet $=$ high reflection and $\mathbf{b}$ ) select cross-sectional b-scan, sliced at green line in $\mathbf{a}\}$.

Figure 16 shows the minimum peak image and a cross-sectional b-scan for cup B. Similarly to cup A, the shape of the base had an impact on the image patterns. A few clusters of higher reflectivity are visible in Fig. 16a, but none provide insight into the nature of the low transmission area in Fig. 13. The b-scan in Fig. 16b is a slice from this area. Interestingly, it shows that between pixels 85 and 35 (left), there are four widely spaced interfaces, separated by 5-10 picoseconds; while between pixels 35 and 15 (right), there are thinner, intermediate layers and the reflections lose intensity at a depth equivalent to the second of the left side's layers. This may be the depth of the metallic layer. 


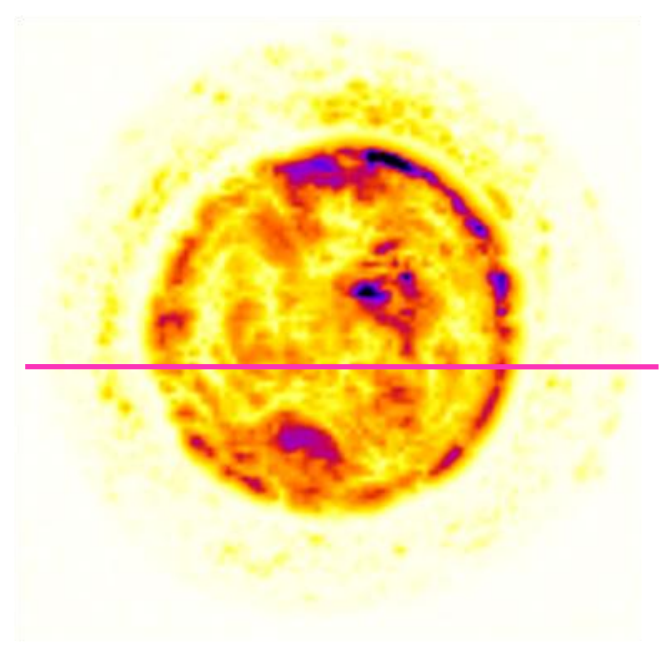

(a)

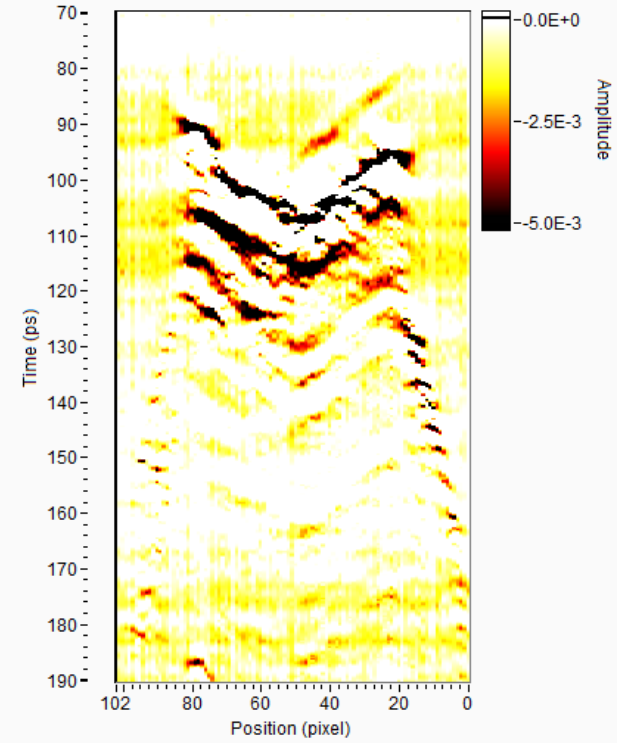

(b)

Fig. 16 a) Terahertz pulse reflection minimum peak image of cup B \{Color scale: White/yellow = low reflection, black/violet $=$ high reflection $\}$ and $b$ ) select cross-sectional b-scan, sliced at pink line in $\mathbf{a}\}$.

Figure 17 shows a reflection image of cup B, calculated by integrating the spectrum of each pixel from 1.0 to $2.0 \mathrm{THz}$. In this spectral range, there is not much variation in the reflectivity of the mineral clusters. However, what is most noticeable is the resemblance of the violet (i.e., the most reflective) region shape to that of the black area in Fig. 13a. This reinforces the notion that the material beneath the surface is not predominantly absorbing, but highly reflective and probably metallic in nature. The dimensions are slightly narrower and better defined in the reflection image, which could be attributed to the smaller beam spot size attributable to the shorter focal length lens used. 


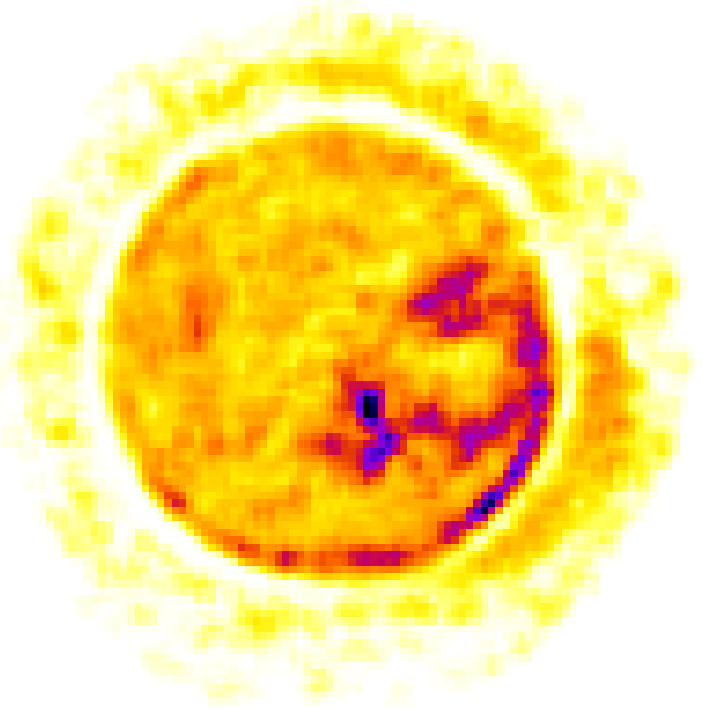

Fig. 17 Frequency-domain power integration between 1.0 and $2.0 \mathrm{THz}$ image of cup B. \{Color scale: White/yellow $=$ low reflection, black/violet $=$ high reflection $\}$

\section{Conclusions}

In this paper we have shown that terahertz pulsed imaging may be useful to archaeologists and conservation scientists for the non-destructive study of artifacts. Compared to other imaging modalities, portable terahertz systems can provide more flexibility in the measurement geometry and scale, and convenience by permitting the system to be taken to the object's location. While the spatial resolution is not as detailed as in x-ray imaging, and there is significant signal loss as the object scale increases, we have demonstrated that terahertz imaging is better able to resolve contrast in the low density components of an ancient Egyptian bird mummy. Additionally, through measurements of ancient Sumerian copper alloy artifacts, we have shown that TPIS can be used for the identification of copper corrosion layers. It can differentiate between metallic and mineralized layers and can qualitatively distinguish different corrosion products.

\section{Acknowledgments}

239 This work was supported by the European Commission's Seventh Framework Program CHARISMA [grant agreement no. 228330] and the Marie Curie Intra-European project TISCH [grant agreement no. 330442].

241 The authors would also like to thank Charles A. Pelizzari-associate professor and director of medical physics in the Department of Radiation and Cellular Oncology at the University of Chicago - and Christian Wietholtan application engineer working for visage Imaging, Inc. and developer of the Amira visualization softwarefor their expertise in X-ray computed tomography and imaging modes.

\section{References}

1. Jackson J B, Bowen J W, Walker G C, Labaune J, Mourou G A, Menu M, Fukunaga K. A Survey of Terahertz Applications in Cultural Heritage Conservation Science. IEEE Transactions on Terahertz Science and Technology, 2011, 1(1): 220-231 doi:10.1109/TTHZ.2011.2159538 Journal of Physical Anthropology, 2010, 142(3): 497-500 doi:10.1002/ajpa.21292 PMID:20544977 
3. Fukunaga K, Cortes E, Cosentino A, Stünkel I, Leona M, Duling I N III, Mininberg D T. Investigating the use of terahertz pulsed time domain reflection imaging for the study of fabric layers of an Egyptian mummy. Journal of the European Optical Society: Rapid Publications, 2011, 6: 11040 doi:10.2971/jeos.2011.11040

254 4. Schmuttenmaer C A. Exploring dynamics in the far-infrared with terahertz spectroscopy. Chemical Reviews, 2004, 104(4): 1759-1779 doi:10.1021/cr020685g PMID:15080711

256 5. Dragoman D, Dragoman M. Terahertz fields and applications. Progress in Quantum Electronics, 2004, 28(1): 1-66

257 doi:10.1016/S0079-6727(03)00058-2

6. Chamberlain JM. Where optics meets electronics: recent progress in decreasing the terahertz gap. Philosophical Transactions Series A, Mathematical, Physical, and Engineering Sciences, 2004, 362:199-211; discussion 212-3 doi:10.1098/rsta.2003.1312

2607 7. Walker G C, Bowen J W, Matthews W, Roychowdhury S, Labaune J, Mourou G, Menu M, Hodder I, Jackson J B. Subsurface terahertz imaging through uneven surfaces: visualizing Neolithic wall paintings in Çatalhöyük. Optics Express, 2013, 21(7): 8126-8134 doi:10.1364/OE.21.008126 PMID:23571902

8. Walker G C, Berry E, Zinovev N N, Fitzgerald A J, Miles R E, Chamberlain J M, Smith M A. Terahertz imaging and international safety guidelines. Proceedings of the Society for Photo-Instrumentation Engineers, 2002, 4682: 683-690 doi: $10.1117 / 12.465614$

9. Kristensen T T, Withayachumnankul W, Jepsen P U, Abbott D. Modeling terahertz heating effects on water. Optics Express, 2010, 18(5): 4727-4739 doi:10.1364/OE.18.004727 PMID:20389486

268 10. Chan W L, Deibel J, Mittleman D M. Imaging with terahertz radiation. Reports on Progress in Physics, 2007, 70(8): 1325-

2691379 doi:10.1088/0034-4885/70/8/R02

11. Adriaens A. European actions to promote and coordinate the use of analytical techniques for cultural heritage studies. TrAC Trends in Analytical Chemistry, 2004, 23(8): 583-586 doi:10.1016/j.trac.2004.07.001

12. Tonouchi M. Galore new applications of terahertz science and technology. Terahertz Science and Technology, 2009, 2: 90101

13. Jepsen P U, Cooke D G, Koch M. Terahertz spectroscopy and imaging - Modern techniques and applications. Laser \& Photonics Reviews, 2011, 5(1): 124-166 doi:10.1002/lpor.201000011

14. Pelizzari C A, Haney C R, Bailleul-LeSuer R, Brown J P, Wietholt C. Challenges in CT scanning of avian mummies. In between heaven and earth: birds in ancient Egypt, Edited by Bailleul-Lesuer R. Chicago: Oriental Institute of the University of Chicago; 2012:109-118.

15. Jackson J B, Mourou G, Labaune J, Menu M. Terahertz pulse imaging of an egyptian bird mummy. In between heaven and earth: birds in ancient Egypt,. 1st edition. Edited by Bailleul-Lesuer R. Chicago: Oriental Institute Museum Publications; 2012:119-122. 16. Luo W, Jin R, Qin Y, Huang F, Wang C. Analysis of the corrosion products of the ancient bronzes excavated from Qiaojiayuan tombs. Applied Physics Research, 2010, 2(2): 156-169

17. Jackson J B, Labaune J, Mourou GA, D'Alessandro L, Whyte A, Menu M. Pulsed terahertz investigation of corroded and mineralized copper alloy historical artifacts. In 2011 International Conference on Infrared, Millimeter, and Terahertz Waves,. Houston, USA: IEEE; 2011:1-2. doi: 10.1109/irmmw-THz.2011.6104844

286 18. Anastasi R F, Madaras E I. Terahertz NDE for under paint corrosion detection and evaluation. AIP Conference Proceedings, 2006, 820: 515-522 doi:10.1063/1.2184571

19. Fuse N, Fukuchi T, Takahashi T, Mizuno M, Fukunaga K. Evaluation of applicability of noncontact analysis methods to detect rust regions in coated steel plates. IEEE Transactions on Terahertz Science and Technology, 2012, 2(2): 242-249 doi:10.1109/TTHZ.2011.2178932

20. Zhao G, Ter Mors M, Wenckebach T, Planken P C M. Terahertz dielectric properties of polystyrene foam. Journal of the Optical Society of America. B, Optical Physics, 2002, 19(6): 1476-1479 doi:10.1364/JOSAB.19.001476

21. Banerjee D, von Spiegel W, Thomson MD, Schabel S, Roskos HG. Diagnosing water content in paper by terahertz radiation. Optics Express, 2008, 16:9060 doi:10.1364/OE.16.009060

22. Roman C, Ichim O, Sarger L, Vigneras V, Mounaix P. Terahertz dielectric characterisation of polymethacrylimide rigid foam: the perfect sheer plate? Electronics Letters, 2004, 40(19): 1167-9 doi:10.1049/el:20045754

23. Fletcher J R, Swift G P, Dai D, Levitt J A, Chamberlain J M. Levitt J a., Chamberlain JM. Propagation of terahertz radiation through random structures: An alternative theoretical approach and experimental validation. Journal of Applied Physics, 2007, 101(1): 013102 doi: $10.1063 / 1.2403860$

300 24. McKnight S W, Stewart K P, Drew H D, Moorjani K. Wavelength-independent anti-interference coating for the far-infrared. 301 Infrared Physics, 1987, 27(5): 327-333 doi:10.1016/0020-0891(87)90074-1 